\title{
Synthesis of New Pyrazolo[1,5-a]pyrimidine, Triazolo $[4,3-a]$ pyrimidine Derivatives, and Thieno[2,3-b]pyridine Derivatives from Sodium 3-(5-Methyl-1-phenyl-1H-pyrazol-4-yl)-3-oxoprop-1-en-1-olate
}

\author{
Abdou O. Abdelhamid and Sobhi M. Gomha \\ Department of Chemistry, Faculty of Science, Cairo University, Giza 12613, Egypt \\ Correspondence should be addressed to Sobhi M. Gomha; s.m.gomha@hotmail.com
}

Received 30 May 2013; Revised 1 October 2013; Accepted 2 October 2013

Academic Editor: Bhimapaka C. Raju

Copyright (C) 2013 A. O. Abdelhamid and S. M. Gomha. This is an open access article distributed under the Creative Commons Attribution License, which permits unrestricted use, distribution, and reproduction in any medium, provided the original work is properly cited.

Condensation of sodium 3-oxo-3-(1-phenyl-1H-pyrazol-4-yl)prop-1-en-1-olate (2) with several heterocyclic amines, cyanoacetamide, cyanothioacetamide, and 2-cyanoacetohydrazide gives pyrazolo $[1,5-a]$ pyrimidines $(\mathbf{5} \mathbf{a}-\mathbf{d})$, pyrido[ $\left[2^{\prime}, 3^{\prime}: 3,4\right]$ pyrazolo[ $[1,5-$ a] pyrimidine (9), benzo[4,5]imidazo[1,2-a]pyrimidine (10), $[1,2,4]$ triazolo[1,5-a]pyrimidine (11), and pyridine derivatives (12-14). Also, thieno[2,3-b]pyridines (15-18) were synthesized via pyridinethione (13) with $\alpha$-halo ketones and $\alpha$-halo ester. Structures of the newly synthesized compounds were elucidated by elemental analysis, spectral data, alternative synthetic routes, and chemical transformation whenever possible.

\section{Introduction}

Several pyrazole derivatives received great attention due to their biological and pharmacological activities not only as potential inhibitors of HIV-1 [1], pesticides [2], fungicides [3], antihypertensive agents [4], and anticancer activity [5], but also as important and useful starting materials for the synthesis of other fused heterocyclic systems. Furthermore, compounds containing the pyrimidine nucleus are of significant biological importance and are used as antibacterial [6-9], antifungal [10, 11], antitumour [12, 13], antiviral [13-17], anti-inflammatory [18, 19], and antihypertensive [20-22] agents. In this work, we synthesized some new pyrazolo[1,5-a]pyrimidine, triazolo[4,3-a]pyrimidine, and thieno $[2,3-b]$ pyridine derivatives.

\section{Experimental}

Melting points were measured on Electrothermal IA 9000 series digital melting point apparatus and are uncorrected.
The IR spectra were recorded in potassium bromide discs on a Pye Unicam SP 3300 and Shimadzu FT IR 8101 PC infrared spectrophotometer. ${ }^{1} \mathrm{H}$ NMR and ${ }^{13} \mathrm{C}$ NMR spectra were recorded in DMSO- $d_{6}$ using a Varian Gemini 300 NMR spectrometer $\left(300 \mathrm{MHz}\right.$ for ${ }^{1} \mathrm{H}$ NMR and $75 \mathrm{MHz}$ for ${ }^{13} \mathrm{C}$ NMR). Mass spectra were recorded on a Shimadzu GCMS-QP1000 EX mass spectrometer at $70 \mathrm{eV}$. Elemental analysis was carried out at the Microanalytical Centre of Cairo University, Giza, Egypt. All reactions were followed by TLC (Silica gel, Merck). 1-(5-Methyl-1-phenyl-1H-pyrazol-4yl)ethanone [23] was prepared as reported in the literature.

2.1. Synthesis of Sodium 3-(5-Methyl-1-phenyl-1H-pyrazol4-yl)-3-oxoprop-1-en-1-olate (2). A mixture of $(0.01 \mathrm{~mol})$ 1-(5-methyl-1-phenyl-1H-pyrazol-4-yl)ethanone 1 (2.0 g, $10 \mathrm{mmol})$ and ethyl formate $(0.74 \mathrm{~g}, 10 \mathrm{mmol})$ was dropped over a solution of sodium methoxide $(0.54 \mathrm{~g}, 10 \mathrm{mmol})$ in $20 \mathrm{~mL}$ dry ether with stirring. The formed solid product was collected and used directly in the following reactions. 


\subsection{Synthesis of $\mathbf{5 a - d}$ and $\mathbf{9 - 1 4}$.}

Method A (general procedure). A mixture of sodium 3-(5methyl-1-phenyl-1H-pyrazol-4-yl)-3-oxoprop-1-en-1-olate (2) $(2.5 \mathrm{~g}, 10 \mathrm{mmol})$ and the appropriate 3 -amino-5-methylpyrazole 3a, 3-amino-5-phenylpyrazole 3b, 3-amino-4cyanopyrazole 3c, 3-amino-4-methyl-5-phenylpyrazole 3d, 4,6-dimethyl-2H-pyrazolo[3,4-b]pyridin-3-amine, 2-aminobenzimidazole, 4H-1,2,4-triazol-3-amine, cyanoacetamide, cyanothioacetamide, and 2-cyanoacetohydrazide $(10 \mathrm{mmol})$ and few drops of acetic acid was grinded for 10-20 min (monitored by TLC). The solid products collected by filtration and recrystallized from the suitable solvent gave the respective products $\mathbf{5 a - d}$ and $\mathbf{9 - 1 4}$ in an excellent yield.

Method B (general procedure). A solution of sodium 3-(5methyl-1-phenyl-1H-pyrazol-4-yl)-3-oxoprop-1-en-1-olate (2) $(2.5 \mathrm{~g}, 10 \mathrm{mmol})$, the appropriate 3-amino-5-methylpyrazole 3a, 3-amino-5-phenylpyrazole $3 \mathbf{b}$, 3-amino-4cyanopyrazole 3c, 3-amino-4-methyl-5-phenylpyrazole 3d, 4,6-dimethyl-2H-pyrazolo[3,4-b]pyridin-3-amine, 2-aminobenzimidazole, 4H-1,2,4-triazol-3-amine, cyanoacetamide, cyanothioacetamide, 2 -cyanoacetohydrazide $(10 \mathrm{mmol})$, and piperidine acetate $(1 \mathrm{~mL})$ in water $(3 \mathrm{~mL})$ was refluxed for $10 \mathrm{~min}$. After completion of the reaction, the hot reaction mixture was neutralized with acetic acid $(1.5 \mathrm{~mL})$ and then cooled and the solid products collected by filtration and recrystallized from the suitable solvent gave products $5 \mathbf{a}-\mathbf{d}$ and 9-14 in a good yield, which were identical in all aspects (m.p., mixed m.p., and spectra) with those obtained from Method A.

2.2.1. 2-Methyl-7-(5-methyl-1-phenyl-1H-pyrazol-4-yl)pyrazolo[1,5-a]pyrimidine (5a). Yield 91\%; yellow crystals; $\mathrm{mp} \mathrm{202-}$ $204^{\circ} \mathrm{C}$ (from acetic acid) (Lit. mp 202-204 $4^{\circ} \mathrm{C}$ [24]).

2.2.2. 7-(5-Methyl-1-phenyl-1H-pyrazol-4-yl)-2-phenylpyrazolo[1,5-a]pyrimidine (5b). Yield 90\%; yellow crystals; m.p. 218$219^{\circ} \mathrm{C}$ (from acetic acid) (Lit. M.p. 216-218 ${ }^{\circ} \mathrm{C}$ [24]).

2.2.3. 7-(5-Methyl-1-phenyl-1H-pyrazol-4-yl)pyrazolo[1,5-a]pyrimidine-3-carbonitrile (5c). Yield $92 \%$; yellow crystals; m.p. $230-232^{\circ} \mathrm{C}$ (from acetic acid); IR (KBr): $v 1594(\mathrm{C}=\mathrm{N})$, $2231(\mathrm{CN}) \mathrm{cm}^{-1} ;{ }^{1} \mathrm{H}$ NMR (DMSO-d $\left.d_{6}\right): \delta 2.56(\mathrm{~s}, 3 \mathrm{H}$, $\left.\mathrm{CH}_{3}\right), 6.38(\mathrm{~d}, 1 \mathrm{H}, J=4 \mathrm{~Hz}$, pyrimidine $\mathrm{H}-5), 6.86(\mathrm{~s}$, $1 \mathrm{H}$, pyrazole $\mathrm{H}-3$ ), $7.28-7.66$ (m, $5 \mathrm{H}$, ArH's), 8.24 (s, $1 \mathrm{H}$, pyrazole $\mathrm{H}-3), 8.71(\mathrm{~d}, 1 \mathrm{H}, J=4 \mathrm{~Hz}$, pyrimidine $\mathrm{H}-4)$; ${ }^{13} \mathrm{C}$ NMR (DMSO- $\left.d_{6}\right): \delta 9.42\left(\mathrm{CH}_{3}\right), 83.11,105.21,111.88$, $112.76,121.87,125.74,128.72,130.11,139.88,140.15,128.55$, 152.37, 155.18; MS $m / z(\%): 301\left(\mathrm{M}^{+}+1,14\right), 300\left(\mathrm{M}^{+}, 41\right)$, 211(32), 84(76), 63(100). Anal. Calcd for $\mathrm{C}_{17} \mathrm{H}_{12} \mathrm{~N}_{6}$ (300.32): C, 67.99; H, 4.03; N, 27.98; Found C, 67.69; H, 4.08; N, $27.68 \%$.

2.2.4. 3-Methyl-7-(5-methyl-1-phenyl-1H-pyrazol-4-yl)-2-phenylpyrazolo[1,5-a]pyrimidine (5d). Yield 90\%; yellow crystals; m.p. $226^{\circ} \mathrm{C}$ (from acetic acid) (Lit. m.p. 226-228 ${ }^{\circ} \mathrm{C}$ [25]).
2.2.5. 8,10-Dimethyl-4-(5-methyl-1-phenyl-1H-pyrazol-4-yl) pyrido $\left[2^{\prime}, 3^{\prime}: 3,4\right]$ pyrazolo[1,5-a]pyrimidine (9). Yield 93\%; yellow crystals; m.p. $290-292^{\circ} \mathrm{C}$ (from acetic acid); IR (KBr): $v 1588(\mathrm{C}=\mathrm{N}) \mathrm{cm}^{-1} ;{ }^{1} \mathrm{H}$ NMR $\left(\mathrm{DMSO}-d_{6}\right): \delta 2.49(\mathrm{~s}, 3 \mathrm{H}$, $\left.\mathrm{CH}_{3}\right), 2.51\left(\mathrm{~s}, 3 \mathrm{H}, \mathrm{CH}_{3}\right), 2.74\left(\mathrm{~s}, 3 \mathrm{H}, \mathrm{CH}_{3}\right), 6.08(\mathrm{~d}, 1 \mathrm{H}$, $J=4 \mathrm{~Hz}$, pyrimidine $\mathrm{H}-5), 6.85$ (s, $1 \mathrm{H}$, pyridine $\mathrm{H}$ ), 7.50-7.59 (m, 5H, ArH's), $8.01(\mathrm{~s}, 1 \mathrm{H}$, pyrazole $\mathrm{H}-3), 8.24(\mathrm{~d}, 1 \mathrm{H}$, $J=4 \mathrm{~Hz}$, pyrimidine $\mathrm{H}-4) ;{ }^{13} \mathrm{C}$ NMR (DMSO- $\left.d_{6}\right): \delta 8.44$ $\left(\mathrm{CH}_{3}\right), 19.21\left(\mathrm{CH}_{3}\right), 21.47\left(\mathrm{CH}_{3}\right), 104.19,110.12,112.85,117.11$, $120.78,122.13,126.88,129.45,133.12,135.48,139.89,140.46$, 148.74, 150.12, 151.78, 165.15; MS $m / z(\%): 355\left(\mathrm{M}^{+}+1,41\right)$, 354( $\left.\mathrm{M}^{+}, 63\right), 262(45), 214(31), 119(28), 77(100)$. Anal. Calcd for $\mathrm{C}_{21} \mathrm{H}_{18} \mathrm{~N}_{6}$ (354.41): C, 71.17; H, 5.12; N, 23.71; Found C, 71.31; H, 5.06; N, $23.56 \%$.

2.2.6. 4-(5-Methyl-1-phenyl-1H-pyrazol-4-yl)benzo[4,5]imidazo[1,2-a]pyrimidine (10). Yield 94\%; yellow crystals; m.p. $203^{\circ} \mathrm{C}$ (from acetic acid) (Lit. m.p. 196-197 ${ }^{\circ} \mathrm{C}[24]$ ).

2.2.7. 7-(5-Methyl-1-phenyl-1H-pyrazol-4-yl)-[1,2,4]triazolo[1,5-a]pyrimidine (11). Yield $94 \%$; yellow crystals; m.p. $180^{\circ} \mathrm{C}$ (from acetic acid) (Lit. m.p. $179-180^{\circ} \mathrm{C}[25]$ ).

2.2.8. 6-(5-Methyl-1-phenyl-1H-pyrazol-4-yl)-2-oxo-1,2-dihydropyridine-3-carbonitrile (12). Yield 91\%; yellow crystals; m.p. $266-268^{\circ} \mathrm{C}$ (from ethanol); IR (KBr): $v 1614(\mathrm{C}=\mathrm{N})$, 2213(CN), 3393(NH) cm ${ }^{-1} ;{ }^{1} \mathrm{H}$ NMR (DMSO- $\left.d_{6}\right): \delta 2.48(\mathrm{~s}$, $\left.3 \mathrm{H}, \mathrm{CH}_{3}\right), 6.97(\mathrm{~d}, 1 \mathrm{H}, J=8.0 \mathrm{~Hz}$, pyridine $\mathrm{H}-5), 7.53-7.73(\mathrm{~m}$, $6 \mathrm{H}$, ArH's and pyrazole $\mathrm{H}-3), 8.18(\mathrm{~d}, 1 \mathrm{H}, J=8.0 \mathrm{~Hz}$, pyridine H-4), 10.21 (s, br., $1 \mathrm{H}, \mathrm{NH}$ ); MS $\mathrm{m} / z$ (\%): $276\left(\mathrm{M}^{+}, 100\right)$, 231(34), 164(36), 118(54), 77(78). Anal. Calcd for $\mathrm{C}_{16} \mathrm{H}_{12} \mathrm{~N}_{4} \mathrm{O}$ (276.29): C, 69.55; H, 4.38; N, 20.28; Found C, 69.45; H, 4.23; N, $20.21 \%$.

2.2.9. 6-(5-Methyl-1-phenyl-1H-pyrazol-4-yl)-2-thioxo-1,2-dihydropyridine-3-carbonitrile (13). Yield 93\%; yellow crystals; m.p. $236-238^{\circ} \mathrm{C}$ (from ethanol); IR ( $\left.\mathrm{KBr}\right): v 1621(\mathrm{C}=\mathrm{N}), 2218$ (CN), $3420(\mathrm{NH}) \mathrm{cm}^{-1} ;{ }^{1} \mathrm{H}$ NMR (DMSO- $\left.d_{6}\right): \delta 2.44(\mathrm{~s}$, $\left.3 \mathrm{H}, \mathrm{CH}_{3}\right), 6.93(\mathrm{~d}, 1 \mathrm{H}, J=7.8 \mathrm{~Hz}$, pyridine $\mathrm{H}-5), 7.52-7.62$ (m, $6 \mathrm{H}$, ArH's and pyrazole $\mathrm{H}-3), 8.12(\mathrm{~d}, 1 \mathrm{H}, J=7.8 \mathrm{~Hz}$, pyridine $\mathrm{H}-4), 14.01(\mathrm{~s}, 1 \mathrm{H}, \mathrm{NH})$; $\mathrm{MS} m / z(\%): 293\left(\mathrm{M}^{+}+1,38\right)$, 292( $\left.\mathrm{M}^{+}, 100\right), 264(16), 181(16), 118(26), 77(82)$. Anal. Calcd for $\mathrm{C}_{16} \mathrm{H}_{12} \mathrm{~N}_{4} \mathrm{~S}$ (292.36): C, 65.73; H, 4.14; N, 19.16; Found C, 65.67; H, 4.10; N, 19.02\%.

2.2.10.1-Amino-6-(5-methyl-1-phenyl-1H-pyrazol-4-yl)-2-oxo1,2-dihydropyridine-3-carbonitrile (14). Yield 92\%; yellow crystals; m.p. $238-140^{\circ} \mathrm{C}$ (from ethanol); IR (KBr): $v 1603$ $(\mathrm{C}=\mathrm{N}), 2214(\mathrm{CN}), 3148,3427\left(\mathrm{NH}_{2}\right) \mathrm{cm}^{-1} ;{ }^{1} \mathrm{H}$ NMR (DMSO$\left.d_{6}\right): \delta 2.52\left(\mathrm{~s}, 3 \mathrm{H}, \mathrm{CH}_{3}\right), 7.37(\mathrm{~d}, 1 \mathrm{H}, J=8.1 \mathrm{~Hz}$, pyridine $\mathrm{H}-$ 5), 6.88 (s, br., $2 \mathrm{H}, \mathrm{NH}_{2}$ ), 7.50-7.56 (m, 5H, ArH's), 7.83 (s, $1 \mathrm{H}$, pyrazole $\mathrm{H}-3), 8.09(\mathrm{~d}, 1 \mathrm{H}, J=8.1 \mathrm{~Hz}$, pyridine $\mathrm{H}-4) ;{ }^{13} \mathrm{C}$ NMR (DMSO- $\left.d_{6}\right): \delta 10.89\left(\mathrm{CH}_{3}\right), 105.22,110.46,113.21,118.24$, $120.58,126.87,128.57,128.89,136.78,139.25,143.22,146.57$, 150.27; MS $m / z(\%): 292\left(\mathrm{M}^{+}+1,38\right), 291\left(\mathrm{M}^{+}, 100\right), 118(11)$, 77(61). Anal. Calcd for $\mathrm{C}_{16} \mathrm{H}_{13} \mathrm{~N}_{5} \mathrm{O}$ (291.31): C, 65.97; H, 4.50; $\mathrm{N}, 24.04$; Found C, 65.78; H, 4.54; N, 23.94\%. 
2.3. 3-Amino-2-substituted-6-(5-methyl-1-phenyl-1H-pyrazol4-yl)thieno[2,3-b]-pyridine Derivatives (15-18).

Method A. A mixture of $13(0.29 \mathrm{~g}, 1 \mathrm{mmol})$ and the appropriate ethyl chloroacetate, chloroacetone, $\omega$-bromoacetophenone or chloroacetonitrile $(1 \mathrm{mmol})$, and 3-5 drops of acetic acid was grinded for $15 \mathrm{~min}$. The resulting solid was collected and recrystallized from ethanol to give the respective compounds, 15-18, in excellent yields.

Method B. A mixture of $\mathbf{1 3}(0.29 \mathrm{~g}, 1 \mathrm{mmol})$ and potassium hydroxide $(0.0 .056 \mathrm{~g}, 1 \mathrm{mmol})$ in $\mathrm{N}, \mathrm{N}$-dimethylformamide $(10 \mathrm{~mL})$ was stirred for $2 \mathrm{~h}$ at room temperature. The appropriate ethyl chloroacetate, chloroacetone, $\omega$-bromoacetophenone, or chloroacetonitrile $(1 \mathrm{mmol}$ each) was added and stirring was continued for $2 \mathrm{~h}$. The resulting solid was collected and recrystallized from ethanol to give 15-18, respectively.

2.3.1. Ethyl 3-Amino-6-(5-methyl-1-phenyl-1H-pyrazol-4-yl)thieno[2,3-b]pyridine-2-carboxylate (15). Yield 88\%; yellow crystals; m.p. $145-146^{\circ} \mathrm{C}$; IR (KBr): $v 1720(\mathrm{C}=\mathrm{O}), 3320,3418$ $\left(\mathrm{NH}_{2}\right) \mathrm{cm}^{-1} ;{ }^{1} \mathrm{H}$ NMR (DMSO- $\left.d_{6}\right): \delta 1.32(\mathrm{t}, J=7.5 \mathrm{~Hz}$, $\left.3 \mathrm{H}, \mathrm{CH}_{3} \mathrm{CH}_{2}\right), 2.49\left(\mathrm{~s}, 3 \mathrm{H}, \mathrm{CH}_{3}\right), 4.16(\mathrm{q}, J=7.5 \mathrm{~Hz}, 2 \mathrm{H}$, $\left.\mathrm{CH}_{2} \mathrm{CH}_{3}\right), 7.82(\mathrm{~d}, \mathrm{H}, J=8.4 \mathrm{~Hz}$, pyridine $\mathrm{H}-5), 7.45-$ $7.82\left(\mathrm{~m}, 7 \mathrm{H}, \mathrm{ArH}\right.$ 's and $\left.\mathrm{NH}_{2}\right), 7.82(\mathrm{~s}, 1 \mathrm{H}$, pyrazole $\mathrm{H}-3)$, $8.48(\mathrm{~d}, 1 \mathrm{H}, J=8.4 \mathrm{~Hz}$, pyridine $\mathrm{H}-4) ;{ }^{13} \mathrm{C}$ NMR (DMSO$\left.d_{6}\right): \delta 11.32,14.58,60.12,105.42,117.45,122.57,126.51,126.89$, $127.74,128.48,132.25,136.75,140.25,143.11,144.87,148.28$, 155.28, 165.78; MS $m / z(\%): 381\left(\mathrm{M}^{+}+1,43\right), 380\left(\mathrm{M}^{+}, 21\right)$, 176(57), 148(57), 102(57), 71(86), 53(100). Anal. Calcd for $\mathrm{C}_{20} \mathrm{H}_{18} \mathrm{~N}_{4} \mathrm{O}_{2} \mathrm{~S}$ (378.45): C, 63.47; H, 4.79; N, 14.80; Found C, $63.49 ; \mathrm{H}, 4.68 ; \mathrm{N}, 14.65 \%$.

2.3.2.1-(3-Amino-6-(5-methyl-1-phenyl-1H-pyrazol-4-yl)thieno[2,3-b]pyridin-2-yl)ethanone (16). Yield 86\%; yellow crystals; m.p. 137-138 ${ }^{\circ} \mathrm{C}$; IR (KBr): $v 1713$ (CO), 3310, 3414 $\left(\mathrm{NH}_{2}\right) \mathrm{cm}^{-1} ;{ }^{1} \mathrm{H}$ NMR (DMSO- $\left.d_{6}\right): \delta 2.12\left(\mathrm{~s}, 3 \mathrm{H}, \mathrm{CH}_{3}\right), 2.38$ (s, $\left.3 \mathrm{H}, \mathrm{CH}_{3}\right), 7.38-7.62\left(\mathrm{~m}, 8 \mathrm{H}, \mathrm{ArH}\right.$ 's, $\mathrm{NH}_{2}$ and pyridine $\mathrm{H}-5), 7.72(\mathrm{~s}, 1 \mathrm{H}$, pyrazole $\mathrm{H}-3), 8.59(\mathrm{~d}, 1 \mathrm{H}, J=8.4 \mathrm{~Hz}$, pyridine $\mathrm{H}-4) ;{ }^{13} \mathrm{C}$ NMR (DMSO- $\left.d_{6}\right): \delta 11.38\left(\mathrm{CH}_{3}\right), 31.25$ $\left(\mathrm{CH}_{3}\right), 117.28,122.58,126.24,126.78,127.34,128.48,132.57$, $136.57,140.38,142.28,144.37,147.81,156.37,191.28$; MS $m / z$ (\%): 349( $\left.\mathrm{M}^{+}+1,21\right), 348\left(\mathrm{M}^{+}, 41\right), 292(32), 149(38), 105(32)$, 77(100), 64(77). Anal. Calcd for $\mathrm{C}_{19} \mathrm{H}_{16} \mathrm{~N}_{4} \mathrm{OS}$ (348.42): C, 65.50; H, 4.63; N, 16.08; Found C, 65.43; H, 4.67; N, $15.87 \%$.

2.3.3. (3-Amino-6-(5-methyl-1-phenyl-1H-pyrazol-4-yl)thien$o[2,3-b]$ pyridin-2-yl)(phenyl)methanone (17). Yield 88\%; yellow crystals; m.p. 127-128 ${ }^{\circ} \mathrm{C}$; IR (KBr): $v 1660$ (CO), 3404 $\left(\mathrm{NH}_{2}\right) \mathrm{cm}^{-1} ;{ }^{1} \mathrm{H}$ NMR (DMSO- $\left.d_{6}\right): \delta 2.48\left(\mathrm{~s}, 3 \mathrm{H}, \mathrm{CH}_{3}\right)$, 7.18-7.58 (m, $14 \mathrm{H}$, ArH's, $\mathrm{NH}_{2}$, pyrazole $\mathrm{H}-3$ and pyridine $\mathrm{H}-5)), 8.54(\mathrm{~d}, 1 \mathrm{H}, J=8.4 \mathrm{~Hz}$, pyridine $\mathrm{H}-4) ; \mathrm{MS}$ $m / z(\%): 411\left(\mathrm{M}^{+}+1,3\right), 410\left(\mathrm{M}^{+}, 10\right), 293(13), 253(18)$, 105(100), 77(81). Anal. Calcd for $\mathrm{C}_{24} \mathrm{H}_{18} \mathrm{~N}_{4} \mathrm{OS}$ (410.49): C, 70.22; H, 4.42; N, 13.65; Found C, 70.18; H, 4.32; N, $13.39 \%$.
2.3.4. 3-Amino-6-(5-methyl-1-phenyl-1H-pyrazol-4-yl)thieno[2,3-b]pyridine-2-carbonitrile (18). Yield 84\%; yellow crystals; m.p. 196-198 ${ }^{\circ} \mathrm{C}$; IR $(\mathrm{KBr}): v 1637(\mathrm{C}=\mathrm{N}), 3200(\mathrm{CN})$, $3343,3410\left(\mathrm{NH}_{2}\right) \mathrm{cm}^{-1} ;{ }^{1} \mathrm{H}$ NMR (DMSO- $\left.d_{6}\right): \delta 2.29(\mathrm{~s}, 3 \mathrm{H}$, $\left.\mathrm{CH}_{3}\right), 7.48-7.60\left(\mathrm{~m}, 7 \mathrm{H}, \mathrm{ArH}\right.$ 's, $\mathrm{NH}_{2}$ and pyrimidine $\left.\mathrm{H}-5\right)$, $7.79(\mathrm{~s}, 1 \mathrm{H}$, pyrazole $\mathrm{H}-3), 8.88(\mathrm{~d}, 1 \mathrm{H}, J=8.4 \mathrm{~Hz}$, pyridine $\mathrm{H}$ 4); MS $m / z(\%): 331\left(\mathrm{M}^{+}, 10\right), 302(17), 179(11), 133(16), 84(100)$, 77(81). Anal. Calcd for $\mathrm{C}_{18} \mathrm{H}_{13} \mathrm{~N}_{5} \mathrm{~S}$ (331.39): C, 65.24; H, 3.95; N, 21.13; Found C, 65.12; H, 3.91; N, 20.83\%.

2.4. 6-(5-Methyl-1-phenyl-1H-pyrazol-4-yl)-2H-pyrazolo[3,4b]pyridin-3-amine (19). To a solution of the thione $13(0.29 \mathrm{~g}$, $0.01 \mathrm{~mol})$ in ethanol $(10 \mathrm{~mL})$ hydrazine hydrate was added $(1 \mathrm{~mL})$ and the mixture was heated under reflux for $12 \mathrm{~h}$. The solution was poured over ice-water mixture and then neutralized by HCl. The solid product was filtered off, dried, and recrystallized from ethanol to afford compound $\mathbf{1 5}$. Yield 72\%; yellow crystals; m.p. $204-206^{\circ} \mathrm{C}$; IR (KBr): $v 1592$ $(\mathrm{C}=\mathrm{N}), 3190,3322\left(\mathrm{NH}_{2}\right.$ and $\left.\mathrm{NH}\right) \mathrm{cm}^{-1} ;{ }^{1} \mathrm{H}$ NMR (DMSO$\left.d_{6}\right): \delta 2.89\left(\mathrm{~s}, 3 \mathrm{H}, \mathrm{CH}_{3}\right), 5.94\left(\mathrm{~s}, \mathrm{br} ., 3 \mathrm{H}, \mathrm{NH}_{2}\right.$ and $\left.\mathrm{NH}\right), 6.92$ (d, $1 \mathrm{H}, J=8.1 \mathrm{~Hz}$, pyridine $\mathrm{H}-4), 7.48-7.56$ (m, 5H, ArH's), $8.14(\mathrm{~s}, 1 \mathrm{H}$, pyrazole $\mathrm{H}-3), 8.48(\mathrm{~d}, 1 \mathrm{H}, J=8.1 \mathrm{~Hz}$, pyridine H-4); MS $m / z(\%): 291\left(\mathrm{M}^{+}+1,18\right), 290\left(\mathrm{M}^{+}, 31\right), 274(52)$, 224(30), 171(28), 118(42), 77(100). Anal. Calcd for $\mathrm{C}_{16} \mathrm{H}_{14} \mathrm{~N}_{6}$ (290.32): C, 66.19; H, 4.86; N, 28.95; Found C, 66.08; H, 4.91; $\mathrm{N}, 28.76 \%$.

2.5. 2,4-Dimethyl-8-(5-methyl-1-phenyl-1H-pyrazol-4-yl)pyrido $\left[2^{\prime}, 3^{\prime}: 3,4\right]$ pyrazolo[1,5-a]pyrimidine (20). To a solution of $19(1.45 \mathrm{~g}, 5 \mathrm{mmol})$ and acetylacetone $(0.5 \mathrm{~g}, 5 \mathrm{mmol})$, in acetic acid $(20 \mathrm{~mL})$ was refluxed for $10 \mathrm{~min}$. The solid product was collected by filtration and recrystallized from acetic acid to afford compound 20. Yield 72\%; yellow crystals; m.p. $206^{\circ} \mathrm{C}$; IR (KBr): $v 1592(\mathrm{C}=\mathrm{N}) \mathrm{cm}^{-1}$; ${ }^{1} \mathrm{H}$ NMR (DMSO- $\left.d_{6}\right)$ : $\delta 2.19\left(\mathrm{~s}, 3 \mathrm{H}, \mathrm{CH}_{3}\right), 2.38\left(\mathrm{~s}, 3 \mathrm{H}, \mathrm{CH}_{3}\right), 2.45\left(\mathrm{~s}, 3 \mathrm{H}, \mathrm{CH}_{3}\right), 6.40$ (s, $1 \mathrm{H}$, pyrimidine $\mathrm{H}-5), 6.50(\mathrm{~d}, 1 \mathrm{H}, J=8 \mathrm{~Hz}$, pyridine $\mathrm{H}-5)$, 7.39-7.50 (m, 5H, ArH's), 8.00 (s, $1 \mathrm{H}$, pyrazole $\mathrm{H}-3), 8.40$ (d, $1 \mathrm{H}, J=12 \mathrm{~Hz}$, pyridine $\mathrm{H}-4) ;{ }^{13} \mathrm{C}$ NMR (DMSO- $\left.d_{6}\right): \delta 10.15$ $\left(\mathrm{CH}_{3}\right), 15.68\left(\mathrm{CH}_{3}\right), 19.36\left(\mathrm{CH}_{3}\right), 104.60,108.10,118.00,119.90$, $122.65,123.38,126.00,129.82,134.65,140.21,142.95,143.42$, $146.00,146.84,161.10,164.44$; MS $m / z(\%): 355\left(\mathrm{M}^{+}+1,1\right)$, 354( $\left.\mathrm{M}^{+}, 3\right), 211(12), 185(31), 157(36), 105(23), 80(100)$. Anal. Calcd for $\mathrm{C}_{21} \mathrm{H}_{18} \mathrm{~N}_{6}$ (354.41): C, 71.17; H, 5.12; N, 23.71; Found C, $71.10 ; \mathrm{H}, 5.19 ; \mathrm{N}, 23.56 \%$.

\section{Results and Discussion}

The starting sodium 3-(5-methyl-1-phenyl-1H-pyrazol-4-yl)3 -oxoprop-1-en-1-olate (2) was prepared via reaction of 1(5-methyl-1-phenyl-1H-pyrazol-4-yl)ethanone [23] (1) with ethyl formate in the presence of sodium methoxide. Thus, condensation of 2 with 5-amino-3-methylpyrazole (3a) in acetic acid containing piperidine acetate or grinding afforded one isolable product formulated as 2-methyl-7-(5-methyl1-phenyl-1H-pyrazol-4-yl)pyrazolo[1,5-a]pyrimidine (5a) in good yield (Scheme 1). ${ }^{1} \mathrm{H}$ NMR for the product revealed the 


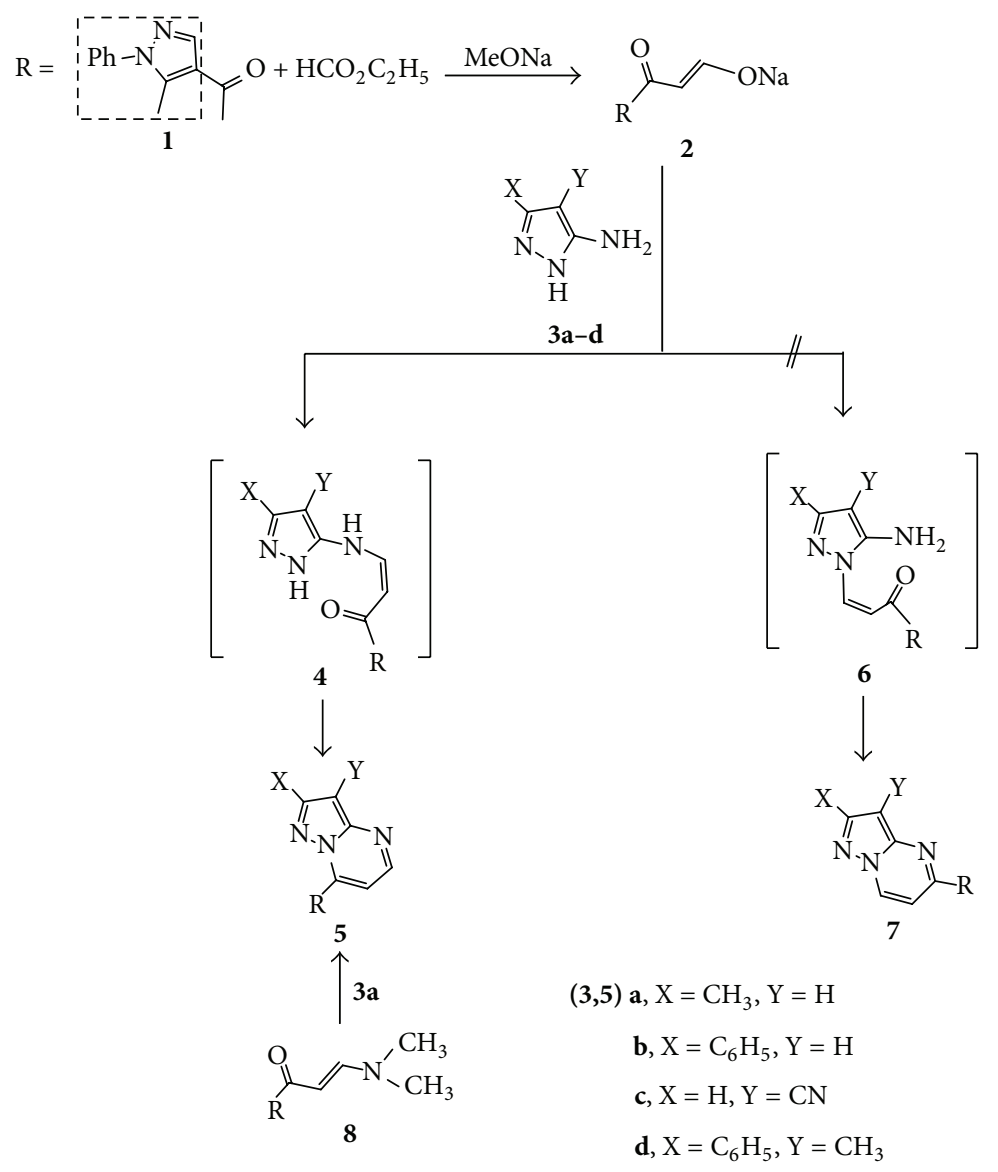

SCHEME 1: Synthesis of pyrazolo[1,5-a]pyrimidines (5a-d).

signals at $\delta=2.61\left(\mathrm{~s}, 3 \mathrm{H}, \mathrm{CH}_{3}\right), 2.69\left(\mathrm{~s}, 3 \mathrm{H}, \mathrm{CH}_{3}\right), 6.36(\mathrm{~d}, 1 \mathrm{H}$, $J=4 \mathrm{~Hz}$, pyrimidine $\mathrm{H}-5), 6.78$ (s, $1 \mathrm{H}$, pyrazole $\mathrm{H}-4), 7.37-$ 7.50 (m, 5H, ArH's), 8.45 (s, 1H, pyrazole H-3), and 8.74 (d, $1 \mathrm{H}, J=4 \mathrm{~Hz}$, pyrimidine $\mathrm{H}-4)$. The reaction seemed to be via the initial nucleophilic attack by the exocyclic amino group at the formyl group, which formed in situ from 1 with water, followed by cyclization and elimination of one molecule of water leading to the formation of the product $\mathbf{5 a}$ (Scheme 1). The suggestion of the formation of the alternative isomeric product $7 \mathbf{a}$ is based on the initial attack of endocyclic amino group at the formyl group for the formation of $7 \mathbf{a}$. As the spectroscopic data above does not allow one to distinguish between possible products $\mathbf{5} \mathbf{a}$ and $7 \mathbf{a}$. The latter suggestion is excluded due to the higher nucleophilicity of the exocyclic primary amino group than the endocyclic amino group. Thus, the mechanism proposed in Scheme 1 seems to be acceptable. In order to establish unambiguously the structure of the product, the crystal structure of a similar previous work has been reported [26-29]. Also, product was obtained by an alternative synthesis route by reaction of 3-(dimethylamino)-1-(5methyl-1-phenyl-1H-pyrazol-4-yl)prop-2-en-1-one (8) [25] with 3a. Analogously, condensation of $\mathbf{3 b}-\mathbf{d}$ with $\mathbf{2}$ gave corresponding 7-substituted pyrazolo[1,5-a]pyrimidines $\mathbf{5 b}$ d, respectively.

Treatment of 2 with the appropriate 4,6-dimethyl- $2 H$ pyrazolo[3,4-b]pyridin-3-amine, 2-aminobenzimidazole, $4 H$-1,2,4-triazol-3-amine, cyanoacetamide and 2-cyanot- hioacetamide, and 2-cyanoacetohydrazide gave 8,10-dimethyl-4-(5-methyl-1-phenyl-1H-pyrazol-4-yl)pyrido $\left[2^{\prime}, 3^{\prime}: 3,4\right]$ pyrazolo[1,5-a]pyrimidine (9), 4-(5-methyl-1-phenyl- $1 H$ pyrazol-4-yl)benzo[4,5]imidazo[1,2-a]pyrimidine (10), 5-(5methyl-1-phenyl-1H-pyrazol-4-yl)-[1,2,4] triazolo[4,3-a]pyrimidine (11), 6(5-methyl-1-phenyl-1H-pyrazol-4-yl)-2-oxo1,2-dihydropyridine-3-carbonitrile (12), 6-(5-methyl-1-phenyl-1H-pyrazol-4-yl)-2-thioxo-1,2-dihydropyridine-3-carbonitrile (13), and 1-amino-6-(5-methyl-1-phenyl-1H-pyrazol4-yl)-2-oxo-1,2-dihydropyridine-3-carbonitrile (14), respectively (Scheme 2). Structures 9-14 were confirmed by elemental analysis, spectral data, and chemical transformation whenever possible.

Thus, compound $\mathbf{1 3}$ reacted with each of ethyl chloroacetate, chloroacetone, $\omega$-bromoacetophenone, chloroacetonitrile and hydrazine hydrate, afforded ethyl 3amino-6-(5-methyl-1-phenyl-1H-pyrazol-4-yl)thieno[2,3- $b$ ] pyridine-2-carboxylate (15), 1-(3-amino-6-(5-methyl-1phenyl-1H-pyrazol-4-yl)thieno[2,3-b] pyridin-2-yl)ethanone (16), (3-amino-6-(5-methyl-1-phenyl-1H-pyrazol-4-yl)thieno[2,3-b] pyridin-2-yl)(phenyl)methanone (17), 3-amino-6(5-methyl-1-phenyl-1H-pyrazol-4-yl)thieno[2,3-b]pyridine2-carbonitrile (18), and 6-(5-methyl-1-phenyl-1H-pyrazol4-yl)-1H-pyrazolo[3,4-b]pyridin-3-amine (19), respectively (Scheme 3).

Structures 15-18 were elucidated by elemental analysis and spectral data. Also, compound 19 reacted with 


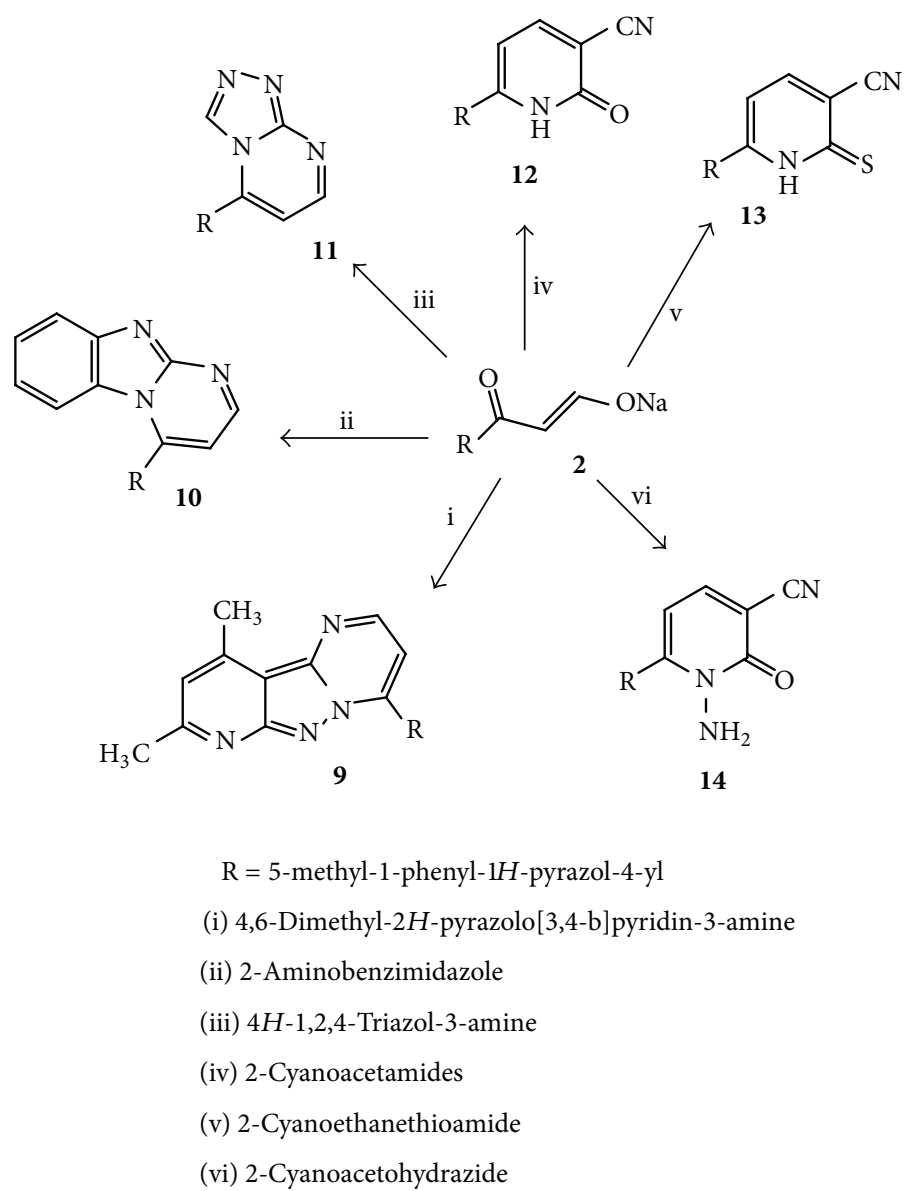

Scheme 2: Synthesis of pyrido $\left[2^{\prime}, 3^{\prime}: 3,4\right]$ pyrazolo[1,5-a]pyrimidine (9), benzoimidazo[1,2-a]pyrimidine (10), triazolo[4,3-a]pyrimidine (11), and pyrimidinones (12-14).<smiles>[R]c1ccc2c(N)c([X])sc2n1</smiles>

15-18<smiles>[R]c1ccc2c(n1)N=N/C2=N\C(C)=C\C</smiles>

20

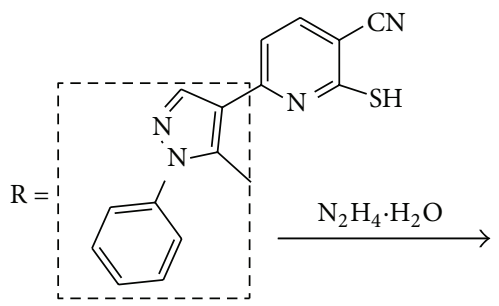

13<smiles>[R]c1ccc2c(N)n[nH]c2n1</smiles>

19

$$
\begin{array}{ll}
\text { 15, } X=\mathrm{CO}_{2} \mathrm{C}_{2} \mathrm{H}_{5} & \text { (i) } \mathrm{ClCH}_{2} \mathrm{CO}_{2} \mathrm{C}_{2} \mathrm{H}_{5} \\
\text { 16, } \mathrm{X}=\mathrm{COCH}_{3} & \text { (ii) } \mathrm{ClCH}_{2} \mathrm{COCH}_{3} \\
\mathbf{1 7}, \mathrm{X}=\mathrm{COC}_{6} \mathrm{H}_{5} & \text { (iii) } \mathrm{BrCH}_{2} \mathrm{COC}_{6} \mathrm{H}_{5} \\
\mathbf{1 8} \mathrm{X}=\mathrm{CN} & \text { (iv) } \mathrm{ClCH}_{2} \mathrm{CN} \\
& \text { (v) }\left(\mathrm{CH}_{3} \mathrm{CO}_{2}\right) \mathrm{CH}_{2}
\end{array}
$$


acetylacetone in acetic acid and gave 2,4-di- methyl-8-(5methyl-1-phenyl-1H-pyrazol-4-yl)pyrido $\left[2^{\prime}, 3^{\prime}: 3,4\right]$ pyrazolo[1,5-a]pyrimidine (20). Structure of $\mathbf{2 0}$ was elucidated via elemental analysis and spectral data.

\section{Conclusion}

In summary, we have developed a simple, efficient procedure for the synthesis of pyrazolo[1,5-a]pyrimidine, pyrido $\left[2^{\prime}, 3^{\prime}: 3,4\right]$ pyrazolo[1,5-a]pyrimidine, $[1,2,4]$ triazolo- $[1,5-$ $a]$ pyrimidine, pyridine, and thieno[2,3-b]pyridines derivatives.

\section{References}

[1] A. M. Kamal El-Dean, A. M. Elkhawaga, S. M. Radwan, and M. M. Ahmed, "Synthesis of some pyridothienopyrazolopyrimidopyrimidine and mercaptomethylpyrazolopyrimidine derivatives," Phosphorus, Sulfur and Silicon and the Related Elements, vol. 184, no. 8, pp. 2034-2048, 2009.

[2] M. Ge, E. Cline, and L. Yang, "A general method for the preparation of 3-acyl-4-cyano-5-amino-pyrazoles," Tetrahedron Letters, vol. 47, no. 32, pp. 5797-5799, 2006.

[3] H. A. El-Sherief, A. M. Mahmoud, and A. A. Ismaiel, "Studies on the synthesis and cyclization reactions of 2-(5-amino-3arylpyrazol-1-yl)-3-methylquinoxalines," Journal of Chemical Research S, no. 9, pp. 322-323, 1997.

[4] A. A. Aly, "Synthesis of polyfunctionally substituted pyrazolonaphthyridine, pentaazanaphthalene, and heptaazaphenanthrene derivatives," Phosphorus, Sulfur and Silicon and the Related Elements, vol. 181, no. 10, pp. 2395-2409, 2006.

[5] A. E. Rashad, M. I. Hegab, R. E. Abdel-Megeid, N. Fathalla, and F. M. E. Abdel-Megeid, "Synthesis and anti-HSV-1 evaluation of some pyrazoles and fused pyrazolopyrimidines," European Journal of Medicinal Chemistry, vol. 44, no. 8, pp. 3285-3292, 2009.

[6] P. Sharma, N. Rane, and V. K. Gurram, "Synthesis and QSAR studies of pyrimido[4,5- $d$ ] pyrimidine-2,5-dione derivatives as potential antimicrobial agents," Bioorganic \& Medicinal Chemistry Letters, vol. 14, no. 16, pp. 4185-4190, 2004.

[7] O. Prakash, V. Bhardwaj, R. Kumar, P. Tyagi, and K. R. Aneja, "Organoiodine (III) mediated synthesis of 3-aryl/hetryl5,7-dimethyl-1,2,4- triazolo[4,3-a]pyrimidines as antibacterial agents," European Journal of Medicinal Chemistry, vol. 39, no. 12, pp. 1073-1077, 2004.

[8] M. Botta, M. Artico, S. Massa et al., "Synthesis, antimicrobial and antiviral activities of isotrimethoprim and some related derivatives," European Journal of Medicinal Chemistry, vol. 27, no. 3, pp. 251-257, 1992.

[9] N. Agarwal, P. Srivastava, S. K. Raghuwanshi et al., "Chloropyrimidines as a new class of antimicrobial agents," Bioorganic \& Medicinal Chemistry, vol. 10, no. 4, pp. 869-874, 2002.

[10] R. A. Forsch, S. F. Queener, and A. Rosowsky, "Preliminary in vitro studies on two potent, water-soluble trimethoprim analogues with exceptional species selectivity against dihydrofolate reductase from Pneumocystis carinii and Mycobacterium avium," Bioorganic \& Medicinal Chemistry Letters, vol. 14, no. 7, pp. 1811-1815, 2004.

[11] N. Agarwal, S. K. Raghuwanshi, D. N. Upadhyay, P. K. Shukla, and V. J. Ram, "Suitably functionalised pyrimidines as potential antimycotic agents," Bioorganic \& Medicinal Chemistry Letters, vol. 10, no. 8, pp. 703-706, 2000.

[12] A. Agarwal, K. Srivastava, S. K. Puri, S. Sinha, and P. M. S. Chauhan, "A small library of trisubstituted pyrimidines as antimalarial and antitubercular agents," Bioorganic \& Medicinal Chemistry Letters, vol. 15, no. 23, pp. 5218-5221, 2005.

[13] T. J. Delia, M. Baumann, and A. Bunker, "Fused pyrimidines. Part 5. Pyrimido[4,5-d]pyrimidine analogues of folic acid," Heterocycles, vol. 35, no. 2, pp. 1397-1410, 1993.

[14] M. T. Cocco, C. Congiu, V. Lilliu, and V. Onnis, "Synthesis and in vitro antitumoral activity of new hydrazinopyrimidine5- carbonitrile derivatives," Bioorganic \& Medicinal Chemistry, vol. 14, no. 2, pp. 366-372, 2006.

[15] V. Malik, P. Singh, and S. Kumar, "Unique chlorine effect in regioselective one-pot synthesis of 1-alkyl-/allyl-3-(ochlorobenzyl) uracils: anti-HIV activity of selected uracil derivatives," Tetrahedron, vol. 62, no. 25, pp. 5944-5951, 2006.

[16] X. Fan, X. Zhang, L. Zhou, K. A. Keith, E. R. Kern, and P. F. Torrence, "A pyrimidine-pyrazolone nucleoside chimera with potent in vitro anti-orthopoxvirus activity, Bioorganic \& Medicinal Chemistry Letters, vol. 16, no. 12, pp. 3224-3228, 2006.

[17] M. Botta, F. Occhionero, R. Nicoletti et al., "Synthesis and biological evaluation of 2-methoxy- and 2-Methylthio-6[(2' -alkylamino)ethyl $]-4(3 \mathrm{H})$-pyrimidinones with anti-rubella virus activity," Bioorganic \& Medicinal Chemistry, vol. 7, no. 9, pp. 1925-1931, 1999.

[18] R. Srivastava, A. Mishra, R. Pratap, D. S. Bhakuni, and R. C. Srimal, "Antithrombotic effect of thiopurinol," Thrombosis Research, vol. 54, no. 6, pp. 741-749, 1989.

[19] H. I. Skulnick, J. H. Ludens, M. G. Wendling et al., "Pyrimidinones. 3. N-substituted 6-phenylpyrimidinones and pyrimidinediones with diuretic/hypotensive and antiinflammatory activity," Journal of Medicinal Chemistry, vol. 29, no. 8, pp. 14991504, 1986.

[20] K. S. Atwal, B. N. Swanson, S. E. Unger et al., "Dihydropyrimidine calcium channel blockers. 3. 3-carbamoyl-4-aryl1,2,3,4-tetrahydro-6-methyl-5-pyrimidinecarboxylic acid esters as orally effective antihypertensive agents," Journal of Medicinal Chemistry, vol. 34, no. 2, pp. 806-811, 1991.

[21] G. C. Rovnyak, K. S. Atwal, A. Hedberg et al., "Dihydropyrimidine calcium channel blockers. 4. Basic 3-substituted4-aryl-1,4-dihydropyrimidine-5-carboxylic acid esters. Potent antihypertensive agents," Journal of Medicinal Chemistry, vol. 35, no. 17, pp. 3254-3263, 1992.

[22] G. J. Grover, S. Dzwonczyk, D. M. McMullen et al., "Pharmacologic profile of the dihydropyrimidine calcium channel blockers SQ 32,547 and SQ 32,946," Journal of Cardiovascular Pharmacology, vol. 26, no. 2, pp. 289-294, 1995.

[23] R. Jakše, J. Svete, B. Stanovnik, and A. Golobič, "Application of alkyl 3-dimethylamino-2-(1H-indol-3-yl)propenoates in the synthesis of 3-heteroarylindoles," Tetrahedron, vol. 60, no. 21, pp. 4601-4608, 2004.

[24] M. R. Shaaban, T. M. A. Eldebss, A. F. Darweesh, and A. M. Farag, "A convenient synthesis of pyrazole-substituted heterocycles," Journal of Chemical Research, vol. 34, no. 1, pp. 8-11, 2010.

[25] M. R. Shaaban, T. M. A. Eldebss, A. F. Darweesh, and A. M. Farag, "Synthesis of novel pyrazolo $[3,4-d]$ pyridazine, pyrido[1,2-a]benzimidazole, pyrimido[1,2-a]benzimidazole and triazolo[4,3-a]pyrimidine derivatives," Journal of Heterocyclic Chemistry, vol. 45, no. 6, pp. 1739-1744, 2008. 
[26] S. A. Ahmed, A. M. Hussein, W. G. M. Hozayen, A. H. H. ElGhandour, and A. O. Abdelhamid, "Synthesis of some pyrazolopyrimidines as purine analogues," Journal of Heterocyclic Chemistry, vol. 44, no. 4, pp. 803-810, 2007.

[27] A. O. Abdelhamid, E. K. A. Abdelall, N. A. Abdel-Riheem, and S. A. Ahmed, "Synthesis and antimicrobial activity of some new 5-arylazothiazole, pyrazolo[1,5-a] pyrimidine, [1,2,4]triazolo[4,3-a]pyrimidine, and pyrimido[1,2-a]benzimidazole derivatives containing the thiazole moiety," Phosphorus, Sulfur and Silicon and the Related Elements, vol. 185, no. 4, pp. 709-718, 2010.

[28] A. O. Abdelhamid, S. A. Shokry, and S. M. Tawfiek, "A new approach for the synthesis of some pyrazolo [5,1-c] triazines and pyrazolo[1,5-a]pyrimidines containing naphtofuran moiety," Journal of Heterocyclic Chemistry, vol. 49, no. 1, pp. 116-124, 2012.

[29] A. O. Abdelhamid, "Convenient synthesis of some new pyrazolo[1,5-a]pyrimidine, pyridine, thieno[2,3-b]pyridine, and isoxazolo $[3,4-d]$ pyridazine derivatives containing benzofuran moiety," Journal of Heterocyclic Chemistry, vol. 46, no. 4, pp. 680-686, 2009. 

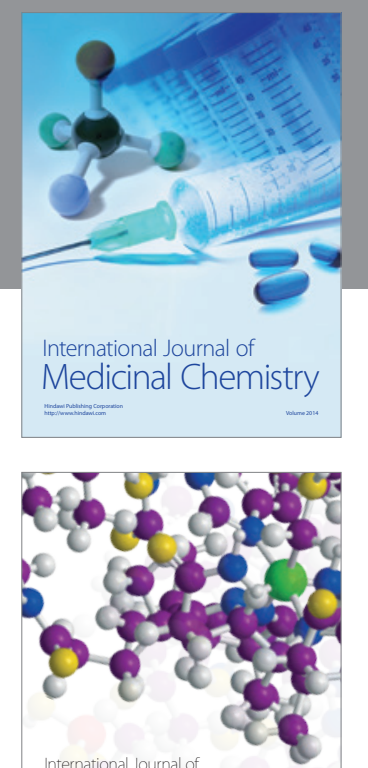

\section{Carbohydrate} Chemistry

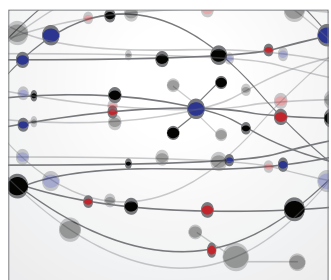

The Scientific World Journal
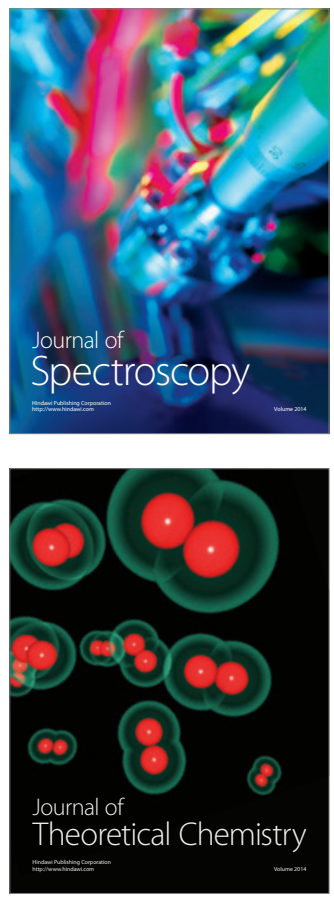
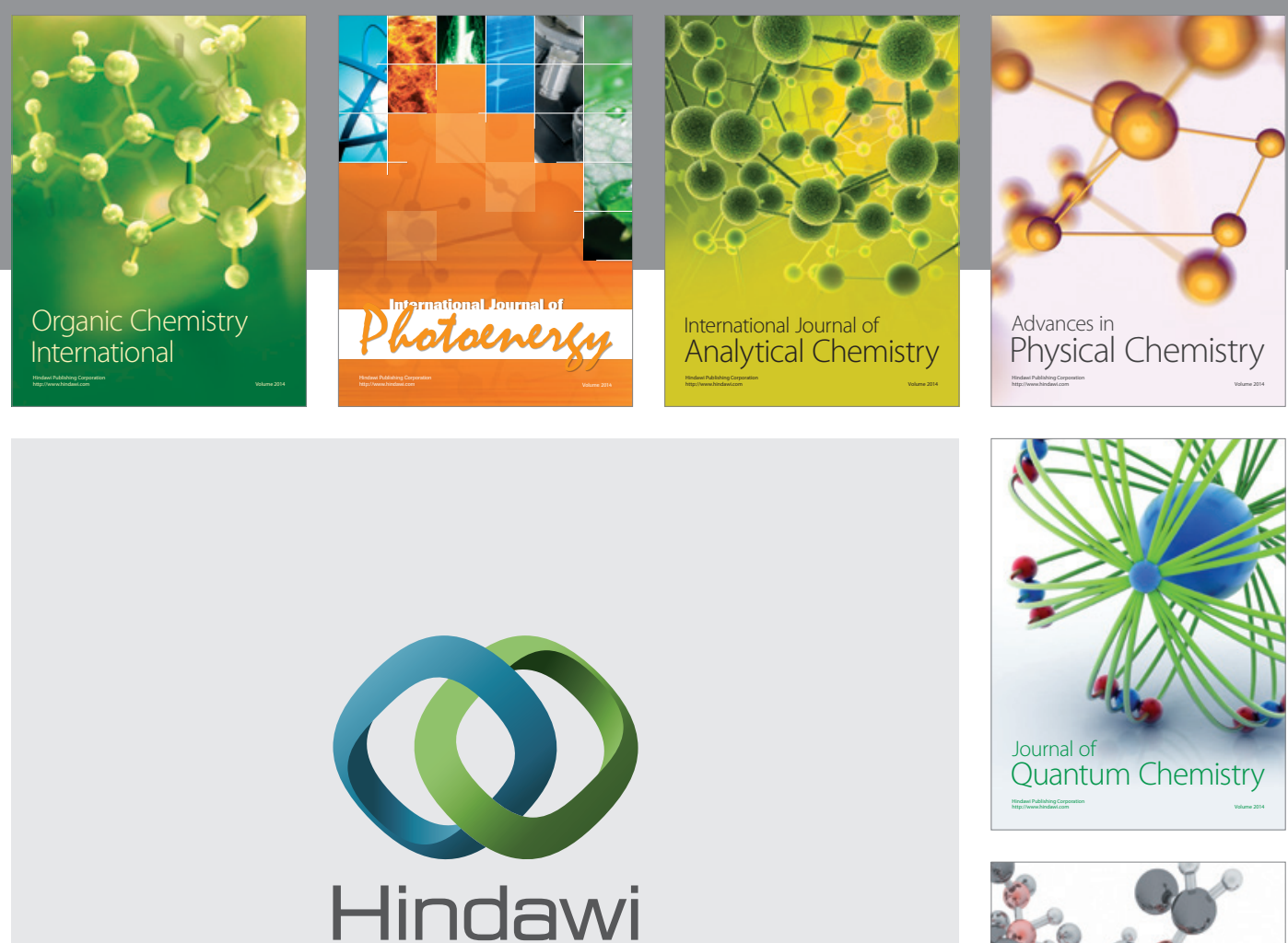

Submit your manuscripts at

http://www.hindawi.com

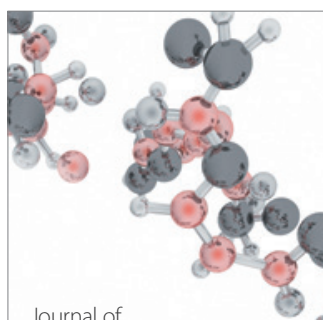

Analytical Methods

in Chemistry

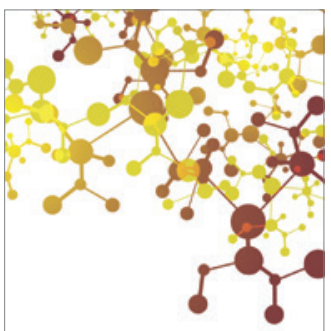

Journal of

Applied Chemistry

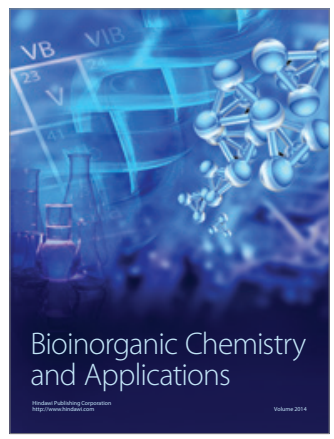

Inorganic Chemistry
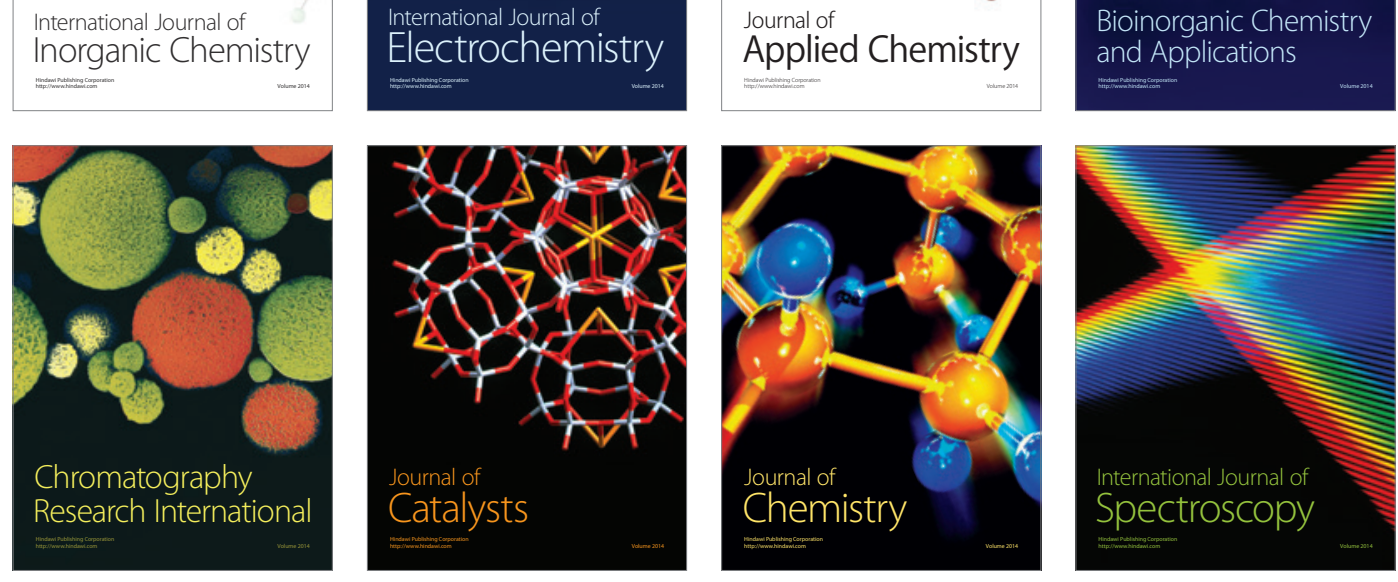